\title{
A Bayesian Perspective on Sparse Regularization for STAP Post-Processing
}

\author{
Jason T. Parker \\ Radar Signal Processing Branch, Sensors Directorate \\ US Air Force Research Laboratory \\ Wright Patterson AFB, OH 45433 \\ Email: jason.parker@wpafb.af.mil
}

\author{
Lee C. Potter \\ Department of Electrical and Computer Engineering \\ The Ohio State University \\ Columbus, $\mathrm{OH} 43210$ \\ Email: potter.36@osu.edu
}

\begin{abstract}
Traditional Space Time Adaptive Processing (STAP) formulations cast the problem as a detection task which results in an optimal decision statistic for a single target in colored Gaussian noise. In the present work, inspired by recent theoretical and algorithmic advances in the field known as compressed sensing, we impose a Laplacian prior on the targets themselves which encourages sparsity in the resulting reconstruction of the angle/Doppler plane. By casting the problem in a Bayesian framework, it becomes readily apparent that sparse regularization can be applied as a post-processing step after the use of a traditional STAP algorithm for clutter estimation. Simulation results demonstrate that this approach allows closely spaced targets to be more easily distinguished.
\end{abstract}

\section{INTRODUCTION}

In order to detect the comparatively weak returns from moving targets, radar systems must suppress returns from the ground and other structures, i.e., clutter, as well as thermal noise in the receiver and active interference sources. White noise in the receiver can only be addressed by increasing the power aperture product of the system. Conversely, clutter and jamming, collectively called interference, exhibit structure that radars can exploit. Space Time Adaptive Processing (STAP) represents the simultaneous adaptive application of both Doppler filtering and spatial beamforming[1], [2] .

By processing a group of pulses known as a coherent processing interval (CPI) from multiple spatial channels, the benefits of both Doppler filtering and beamforming can be realized. In addition, STAP allows the suppression of interference which exhibits structure in the joint-space time domain that neither technique could individually address. While much of the early work in STAP focused on the simplest case of side-looking uniform linear arrays (ULAs) operating monostatically, STAP techniques have also been applied to bistatic systems, conformal arrays, space-based systems, and other applications [3].

Recently, an increasing interest in compressed sensing[4], [5], [6] along with a history of sparsity and randomization techniques in radar [7] has compelled several authors to consider applying CS ideas to moving target indication (MTI) and STAP problems [8], [9], [10]. The core notion in CS is to regularize a linear inverse problem by including prior knowledge that the signal of interest is sparse. The signals of interest in MTI are not sparse, because they contain large high-energy clutter components that span a wide range in angle/Doppler.

However, the targets of interest are sparse in the angle/Doppler plane, often occupying just a few cells. Existing approaches have tried to address this dichotomy by reconstructing the clutter as part of the target signal using various ad hoc approaches to deal with the large amplitude clutter components (see Section III). While this approach offers potential gains in processing performance and will likely be the subject of extensive future research, herein we propose a simpler scheme exploiting sparseness of targets in STAP.

Specifically, casting the problem in a Bayesian framework reveals that clutter whitening can first be accomplished using any traditional STAP technique. Subsequently, sparse regularization can be used to significantly improve the quality of the target estimates. Simulation results demonstrate that the sparse regularization approach can resolve closely spaced targets that are difficult to distinguish using traditional STAP. All results will be computed using clairvoyant clutter knowledge, since the goal of this paper is to demonstrate the utility of sparse regularization as a post-processing technique, rather than a clutter estimation tool.

The paper is organized as follows: Section II describes the signal model, Section III briefly reviews prior work, Section IV describes the proposed approach, and Section V provides some simulated examples. Finally, conclusions and directions for further research are provided in Section VI.

\section{Signal Model}

The techniques in this paper can be easily applied to conformal arrays, bistatic geometries, and other problems of interest. For ease of exposition, the development will be restricted to a monostatic ULA. Consider a ULA consisting of $J$ channels spaced equally at $d$ meters apart. A CPI for this system will consist of data collected over $K$ slow time pulses with a sampling period of $T$ seconds and $L$ fast time range bins ${ }^{1}$. We shall assume that the system is narrowband, i.e., the

\footnotetext{
${ }^{1}$ Slow time refers to the relatively long intervals between successive pulses from a coherent radar. Fast time refers to the time scale at which the electromagnetic pulse travels across the scene of interest, which is the same as range up to a scale factor.
} 
bandwidth $B<<f_{c}$, where $f_{c}=\frac{c}{\lambda}$ is the center frequency ${ }^{2}$ and that pulse compression has already been performed. In addition, target motion during a given pulse will be neglected ${ }^{3}$.

Often, a CPI of radar data is organized into a data cube, where the three dimensions are channels, pulses, and fast time. Since our goal is to highlight the underlying linear problem and the applicability of a CS approach, we will immediately consider space-time snapshots, i.e., the vectorization of the $2 \mathrm{D}$ data cube slice at a particular range. Specifically, the data for pulse $k$ at range $l$ will be denoted as $y_{k, l} \in \mathbb{C}^{J}$, while the space-time snapshot at range $l$ will be denoted $y_{l} \in \mathbb{C}^{J K}$ where the data has been organized pulse-wise, i.e.,

$$
y_{l}=\left[\begin{array}{llll}
y_{1, l}^{T} & y_{2, l}^{T} & \cdots & y_{K, l}^{T}
\end{array}\right]^{T} .
$$

The data matrix containing the complete set of complex samples for the entire CPI is then given as $Y \in \mathbb{C}^{J K \times L}$, where

$$
Y=\left[\begin{array}{llll}
y_{1} & y_{2} & \ldots & y_{L}
\end{array}\right] .
$$

At a given range, the response of the array over the CPI to a point target can be characterized with a space-time steering vector. First, consider the response of the $J$ spatial channels to a target response arriving at elevation angle $\theta$ and azimuth angle $\phi$. Since the array is linear in the azimuth plane, there is a conical ambiguity in the arrival direction of a given signal characterized by the cone angle $\theta_{c}=\cos ^{-1}(\cos \theta \sin \phi)$. The spatial frequency observed by the array for a given cone angle is then

$$
f_{s}=\frac{d}{\lambda} \cos \theta_{c} .
$$

The spatial steering vector for a given cone angle is then $a_{s} \in$ $\mathbb{C}^{J}$ given by

$a_{s}\left(f_{s}\right)=\left[\begin{array}{llll}1 & \exp \left(j 2 \pi f_{s}\right) & \ldots & \exp \left(j 2 \pi(J-1) f_{s}\right)\end{array}\right]^{T}$,

where we have selected the first element as the zero-phase reference for the array. Similarly, we can define the normalized Doppler frequency as

$$
f_{d}=\frac{2 v T}{\lambda}
$$

where $v$ is the velocity of the target. The temporal steering vector describing the response of a single element across $K$ time samples to a target at normalized Doppler $f_{d}$ is then given as the length $K$ vector

$a_{t}\left(f_{d}\right)=\left[\begin{array}{llll}1 & \exp \left(j 2 \pi f_{d}\right) & \ldots & \exp \left(j 2 \pi(K-1) f_{d}\right)\end{array}\right]^{T}$.

The combined space time steering vector for a target is then given as the Kronecker product of the temporal and spatial steering vectors, i.e.,

$$
a\left(f_{s}, f_{d}\right)=a_{t}\left(f_{d}\right) \otimes a_{s}\left(f_{s}\right)
$$

\footnotetext{
${ }^{2}$ This assumption allows time delays to be well approximated as phase shifts, which creates a correspondence between target velocity and the output bins of an FFT with respect to slow time.

${ }^{3}$ This so called stop-and-hop approximation is very reasonable for the short pulses associated with MTI platforms [11].
}

Let us discretize the frequency variables into $N_{s} \geq J$ spatial frequency bins and $N_{d} \geq K$ Doppler frequency bins spaced uniformly across the allowed ranges for each variable to obtain $N=N_{s} N_{d}$ unique steering vectors. We can organize the resulting steering vectors into a matrix $A \in \mathbb{C}^{J K \times N}$. Neglecting range ambiguities, we can define the scene reflectivity as a function of $f_{s}$ and $f_{d}$ at a given range as $x_{l} \in \mathbb{C}^{N}$. We then obtain the linear relationship between the collected data and the scene of interest as

$$
\begin{aligned}
& y_{l}=A x_{l}+q_{l} \\
& Y=A X+Q,
\end{aligned}
$$

where $X \in C^{N \times L}$ is the collection of the $L$ scene vectors $\left\{x_{l}\right\}$. We have used $q_{l}$ to denote the noise plus interference in the $l$ th range bin, and $Q$ to represent the matrix of these disturbances. Throughout the remainder of the paper our results will focus on analyzing only a single range bin with known clutter statistics. Straddling losses caused by discretization, along with array calibration errors and other similar effects, can be captured by including an unknown perturbation in the matrix $A$. Preliminary theoretical results suggest that CS approaches are to some extent robust against these modeling errors [12], [13].

\section{PRIOR WORK}

\section{A. Sparse Regularization}

A distinction should be made between compressed sensing (CS), which specifically involves random matrices and performance guarantees based on the restricted isometry property (RIP), mutual coherence, or similar notions [14] and sparse regularization, which simply includes a sparsity-inducing regularization term. The two are closely related and utilize many of the same tools. In particular, both often lead to problem formulations that require the solution of the so called Basis Pursuit Denoising (BPDN) problem which takes the form

$$
\underset{x}{\arg \min }\|A x-y\|_{2}^{2}+\lambda\|x\|_{1},
$$

where $\|x\|_{1}$ is the $\ell_{1}$ or "taxicab" norm, given by the sum of the absolute values of the elements of the vector $x$.

Omitting details beyond the scope of this paper, this formulation is selected because it is represents a convex relaxation [15] of the problem of enforcing maximal sparsity on the signal $x$, which is an NP-hard problem. While this problem can be cast as a second order cone program and solved with interior point methods [4], this approach is often computationally intractable for large scale problems like those encountered in radar. A variety of algorithms have been proposed to attack this and similar problems including gradient based methods [16], [17], [18], [19], greedy methods [20], [21], [22], and re-reweighting approaches that solve a sequence of $\ell_{2}$ problems to approximate $\ell_{1}$ minimization [23]. 


\section{B. Applications to STAP}

At the time of this publication, the authors are only aware of three references describing the application of sparse regularization to STAP. The first of these papers [9] addresses the problem of clutter by applying a mask to the signal in the angle/Doppler domain before penalizing. Specifically, they minimize

$$
J(x)=\|y-A x\|_{2}^{2}+\lambda\|M x\|_{1},
$$

where $M$ is a mask that zeroes out the components of the signal that correspond to the clutter ridge. Given knowledge of the clutter ridge location, this technique is shown to outperform the sample matrix inverse (SMI). The results use the convex optimization algorithm known as FISTA [17].

The second paper [10] does not deal with the clutter ridge through optimization of a modified cost function. Instead of masking off the clutter ridge, the entire scene is reconstructed using a CS approach, and then an attempt is made to identify and zero out the clutter component. Three algorithms are presented that carry out this procedure, including variants that attempt to exploit available training data. The paper avoids using prior knowledge of the clutter ridge location at the expense of requiring the signal to be sparse in the angle/Doppler domain with the clutter included. The final paper [8] similarly attempts to reconstruct the clutter and target signals together.

This paper offers a formulation in a Bayesian framework using simple priors that allows sparse reconstruction algorithms to be leveraged in concert with existing STAP algorithms to improve reconstruction quality. The algorithm relies on a traditional STAP algorithm for clutter whitening, which differs from the single snapshot approaches cited above.

\section{Sparse Regularization Post-Processing}

To cast the estimation of $x_{l}$ in a Bayesian framework, we must adopt priors on the signal and disturbance. First, we will adopt a Laplacian prior on the unknown signal $x_{l}$ and assume that the noise $q_{l}$ is circular Gaussian with known covariance $\Sigma_{l}$, i.e.,

$$
\begin{aligned}
q_{l} & \sim \mathcal{C N}\left(0, \Sigma_{l}\right) \\
p\left(x_{l}\right) & \propto \exp \left\{-\frac{\lambda}{2}\left\|x_{l}\right\|_{1}\right\},
\end{aligned}
$$

where the normalization constant on $p\left(x_{l}\right)$ is omitted for simplicity. We can then find the MAP (maximum a posteriori) estimate easily as

$$
\begin{aligned}
\hat{x}_{l} & =\underset{x}{\arg \max } p\left(x \mid y_{l}\right) \\
& =\underset{x}{\arg \max } \frac{p\left(y_{l} \mid x\right) p(x)}{p(y)} \\
& =\underset{x}{\arg \max } p\left(y_{l} \mid x\right) p(x) \\
& =\underset{x}{\arg \max } \exp \left\{-\frac{1}{2}\left\|A x-y_{l}\right\|_{\Sigma_{l}}^{2}\right\} \exp \left\{-\frac{\lambda}{2}\|x\|_{1}\right\} \\
& =\underset{x}{\arg \min }\left\|A x-y_{l}\right\|_{\Sigma_{l}}^{2}+\lambda\|x\|_{1},
\end{aligned}
$$

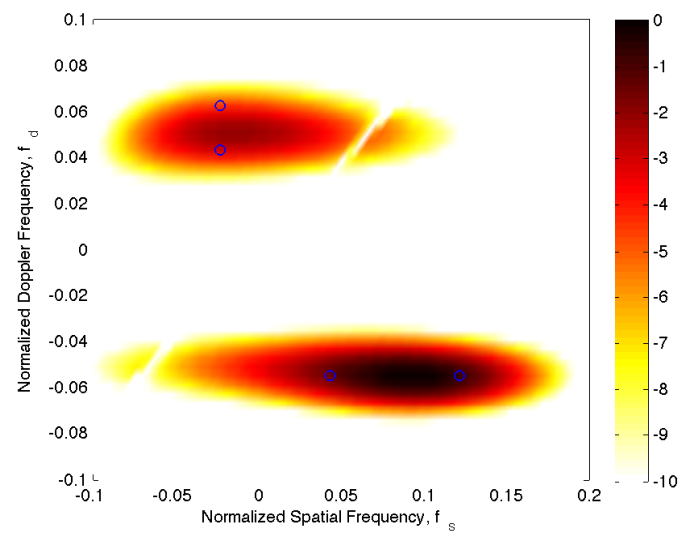

Fig. 1. NMF test statistic results for one clutter realization. The two pairs of closely spaced targets are blurred together. The results are provided on a normalized $\mathrm{dB}$ scale, and the true target positions are marked with blue circles.

where $\|x\|_{\Sigma_{l}}^{2}=x^{H} \Sigma_{l}^{-1} x$. The resulting optimization problem is precisely what we would expect given the colored Gaussian noise prior. Since $\Sigma_{l}$ is a covariance matrix, and hence positive definite and symmetric, the problem is convex and solvable with a variety of techniques. In fact, we can factor the inverse of the covariance using the Cholesky decomposition as $\Sigma^{-1}=$ $R^{H} R$ to obtain

$$
\begin{aligned}
\hat{x}_{l} & =\underset{x}{\arg \min }\left\|A x-y_{l}\right\|_{\Sigma_{l}}^{2}+\lambda\|x\|_{1} \\
& =\underset{x}{\arg \min }\left\|R A x-R y_{l}\right\|_{2}^{2}+\lambda\|x\|_{1} \\
& =\underset{x}{\arg \min }\left\|\bar{A} x-\bar{y}_{l}\right\|_{2}^{2}+\lambda\|x\|_{1},
\end{aligned}
$$

where $\bar{A}=R A$ and $\bar{y}_{l}=R y_{l}$. With this transformation, the problem is now in the standard BPDN form and can be solved using any of the approaches referenced in Section III.

This simple calculation resulting in Eqn (1) is very illuminating. After whitening the clutter using any standard STAP algorithm, a sparse regularization post-procesing step can be applied to capitalize on the sparsity of targets in most MTI scenarios. This post-processing can significantly improve the quality of the output reconstruction without requiring modification to the clutter covariance estimation technique.

\section{Simulation Results}

An airborne, side-looking L-band radar platform with an $J=8$ channel ULA was simulated using the SMS-MBS software package [24]. A CPI of $K=32$ pulses was processed using clairvoyant knowledge of the clutter covariance matrix. Two pairs of targets were placed near the clutter ridge near the pointing angle of the transmit beam. One pair has the same normalized Doppler frequency, while the other shares the same normalized spatial frequency.

The results in Figure 1 show the performance of the normalized matched filter (NMF). This test statistic is calculated 


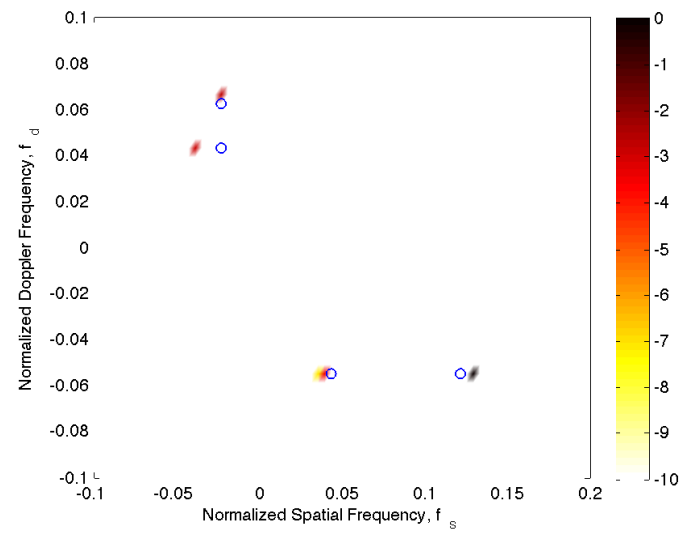

Fig. 2. Reconstruction using FISTA for sparse regularization post-procesisng for one clutter realization. Both pairs of closely spaced targets are now clearly resolved. The results are provided on a normalized $\mathrm{dB}$ scale, and the true target positions are marked with blue circles.

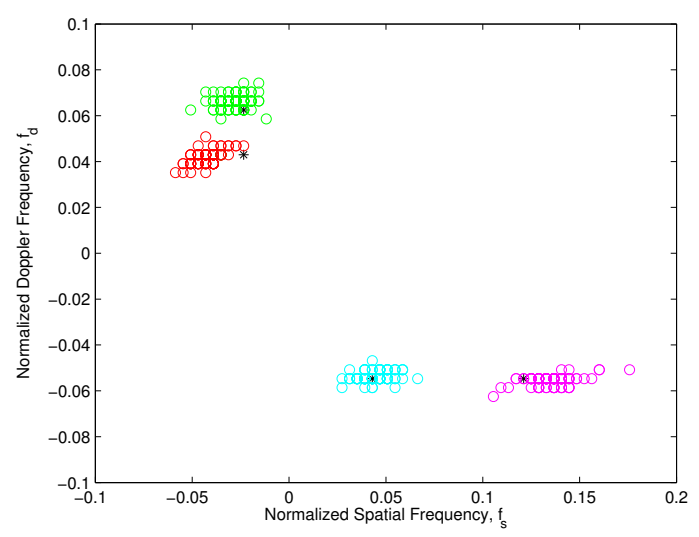

Fig. 3. The black stars mark the true target locations. The clusters of colored circles represent the four strongest local maxima in the FISTA reconstruction for each of 100 clutter realizations. The target clusters are well separated, although some bias in location estimation is observed.

as

$$
\hat{x}_{N M F}\left(f_{s}, f_{d}\right)=\frac{\left|a\left(f_{s}, f_{d}\right)^{H} \Sigma^{-1} y\right|^{2}}{\left(a\left(f_{s}, f_{d}\right)^{H} \Sigma^{-1} a\left(f_{s}, f_{d}\right)\right)\left(y^{H \Sigma^{-1}} y\right)},
$$

which represents matched filtering after optimal clutter whitening. The denominator is included to provide the constant false alarm rate (CFAR) property [25]. While $\hat{x}_{N M F}$ is the optimal test statistic for a single target, the resulting image blurs together each pair of closely spaced targets. Local peak detection demonstrates that only a single local maximum appears at the location of each target pair.

In contrast, the result using the proposed approach shown in Figure 2 clearly separates the targets and provides a cleaner image, requiring no further processing. The estimated locations of the targets are somewhat biased in the angle/Doppler plane, but two targets in approximately the correct configuration are still detected in each pair.

To assess the variance and bias of the target location estimates in the angle/Doppler plane, a series of 100 trials were conducted with random realizations of the disturbance signal. Figure 3 plots the four largest local maxima from the reconstruction for each realization. The clusters of estimated target locations are well separated. The bias in the estimate of the lower Doppler frequency target in the pair arriving at $f_{s}=-0.234$ is likely due to a combination of the proximity to the clutter ridge and interference effects between the two targets.

The regularization parameter $\lambda$ was chosen in an ad hoc manner for this simple test. As suggested in [9], the FISTA algorithm was used to solve Eqn (1) to take advantage of its simplicity and guaranteed quadratic rate of convergence. As an aside, the FISTA algorithm is a majorization-minimization approach with close ties to the algorithm proposed in [18].

\section{CONCLUSION}

The plethora of recent developments in compressed sensing offers a variety of algorithms for solving the BPDN problem. The present work casts the STAP problem in a Bayesian framework with a Laplacian prior on the target reflectivity as a function of angle and Doppler. This formulation allows sparse regularization to be applied as a post-procesing step after clutter whitening using any standard STAP technique. The provided simulation results demonstrate that the proposed approach can significantly enhance the quality of angle/Doppler reconstructions. In particular, the resolvability of closely spaced targets is enhanced, even compared to the optimal single target STAP processor with clairvoyant clutter knowledge. Future work will include extending this Bayesian framework to handle other clutter and target priors and incorporating simultaneous estimation of the clutter statistics into the proposed sparse regularization scheme.

\section{ACKNOWLEDGMENT}

The authors would like to thank Professor Ivan Selesnick and Dr. Braham Himed for valuable discussions about their ongoing work in this area. We would also like to thank Dr. Murali Rangaswamy for reading an early version of this manuscript. Finally, the first author is supported by an AFOSR lab task under the direction of Dr. Arje Nachman.

\section{REFERENCES}

[1] J. Guerci, Space-time adaptive processing for radar. Artech House, 2003.

[2] R. Klemm, Applications of space-time adaptive processing. Inspec/IEE, 2004.

[3] W. Melvin, "A STAP Overview," IEEE Aerospace and Electronic Systems Magazine, vol. 19, no. 1 Part 2, pp. 19-35, 2004.

[4] E. Candès, J. Romberg, and T. Tao, "Stable signal recovery from incomplete and inaccurate measurements," Communications on pure and applied mathematics, vol. 59, no. 8, pp. 1207-1223, 2006.

[5] D. L. Donoho, "Compressed sensing," IEEE Transactions on Information Theory, vol. 52, no. 4, pp. 1289-1306, April 2006.

[6] E. Candes, "Compressive sampling," Proceedings of the International Congress of Mathematicians, Madrid, Spain, vol. 3, pp. 1433-1452, 2006.

[7] L. C. Potter, E. Ertin, J. T. Parker, and M. Cetin, "Sparsity and compressed sensing in radar imaging," To Appear in the Proceedings of the IEEE Special Issue on Applications of Compressive Sensing, 2010. 
[8] S. Maria and J.-J. Fuchs, "Application of the global matched filter to STAP data an efficient algorithmic approach," in Proc. IEEE International Conference on Acoustics, Speech and Signal Processing ICASSP 2006, vol. 4, May 14-19, 2006, p. IV.

[9] I. W. Selesnick, S. U. Pillai, K. Y. Li, and B. Himed, "Angle-doppler processing using sparse regularization," ICASSP, 2010.

[10] H. Zhang, G. Li, and H. Meng, "A class of novel STAP algorithms using sparse recovery technique," Arxiv preprint arXiv:0904.1313, 2009.

[11] M. Richards, Fundamentals of radar signal processing. McGraw-Hill, 2005.

[12] M. A. Herman and T. Strohmer, "General deviants: An analysis of perturbations in compressed sensing," Accepted IEEE Journal of Selected Topics in Signal Processing: Special Issue on Compressive Sensing, 2010.

[13] C. D. Austin, R. L. Moses, J. N. Ash, and E. Ertin, "On the relation between sparse reconstruction and parameter estimation with order order selection," To Appear IEEE Journal of Selected Topics in Signal Processing, 2010.

[14] E. J. Candes and M. B. Wakin, "An introduction to compressive sampling," IEEE Signal Processing Magazine, vol. 25, no. 2, pp. 21-30, Mar. 2008.

[15] J. A. Tropp, "Just relax: convex programming methods for identifying sparse signals in noise," IEEE Transactions on Information Theory, vol. 52, no. 3, pp. 1030-1051, March 2006.

[16] M. A. T. Figueiredo, R. D. Nowak, and S. J. Wright, "Gradient projection for sparse reconstruction: Application to compressed sensing and other inverse problems," IEEE Journal of Selected Topics in Signal Processing, vol. 1, no. 4, pp. 586-597, Dec. 2007.

[17] A. Beck and M. Teboulle, "A fast iterative shrinkage-thresholding algorithm for linear inverse problems," SIAM Journal on Imaging Sciences, vol. 2, no. 1, pp. 183-202, 2009.

[18] T. J. Kragh and A. A. Kharbouch, "Monotonic iterative algorithms for SAR image restoration," in Proc. IEEE International Conference on Image Processing, 8-11 Oct. 2006, pp. 645-648.

[19] M. Cetin and W. C. Karl, "Feature-enhanced synthetic aperture radar image formation based on nonquadratic regularization," IEEE Transactions on Image Processing, vol. 10, no. 4, pp. 623-631, April 2001.

[20] D. Needell and J. A. Tropp, "CoSaMP: Iterative signal recovery from incomplete and inaccurate samples," Applied Computational Harmonic Analysis, vol. 26, pp. 301-321, 2008.

[21] D. L. Donoho, Y. Tsaig, I. Drori, and J.-C. Starck, "Sparse solution of underdetermined linear equations by stagewise orthogonal matching pursuit," Dept. of Statistics, Stanford University, Stanford, CA, Tech. Rep. 2006-02, 2006, (software available at http://sparselab.stanford.edu/).

[22] J. A. Tropp and A. C. Gilbert, "Signal recovery from random measurements via orthogonal matching pursuit," IEEE Transactions on Information Theory, vol. 53, no. 12, pp. 4655-4666, Dec. 2007.

[23] I. F. Gorodnitsky and B. D. Rao, "Sparse signal reconstruction from limited data using focuss: a re-weighted minimum norm algorithm," IEEE Transactions on Signal Processing, vol. 45, no. 3, pp. 600-616, March 1997.

[24] Y. Zhang and A. Hajjari, "Bistatic space-time adaptive processing (STAP) for airborne/spaceborne applications - signal modeling and simulation tool for multichannel bistatic systems (SMS-MBS)," Air Force Research Laboratory Final Technical Report AFRL-SN-RS-TR2002-201, Aug 2002.

[25] J. H. Michels, B. Himed, and M. Rangaswamy, "Performance of STAP tests in gaussian and compound-gaussian clutter," Digital Signal Processing, vol. 10, no. 4, pp. $309-324,2000$. 